\title{
Mucin depletion in inflammatory bowel disease
}

\author{
D A McCormick, L W L Horton, A S Mee
}

Abstract

The mucin and gland content of 26 rectal biopsy specimens-five normal specimens, 10 from patients with ulcerative colitis, and 11 from patients with Crohn's disease-were measured using a Quantimet image analyser. There was significantly less mucin in the groups with ulcerative colitis compared with either those with Crohn's disease or the normal controls. The difference in the gland content between the groups with ulcerative colitis and Crohn's disease and between the group with Crohn's disease and the normal controls did not reach significance.

The results suggest that it is worth while assessing the mucin content of rectal biopsy specimens from patients with inflammatory bowel disease. In routine practice this assessment can be made by eye using a suitably stained section.

The distinction between Crohn's disease and ulcerative colitis on rectal biopsy specimens is not merely an academic pursuit. The management of the patient including the advisability, type, and extent of surgical intervention are influenced by the histological diagnosis. ${ }^{1}$ These two diseases can be easily distinguished if granulomata are seen, but the incidence of granulomata in surgical bowel resections for Crohn's disease is only $60 \%{ }^{2}$ and in rectal biopsy specimens the incidences have ranged from 0 to $28 \%$ depending on the population group studied. ${ }^{134}$ Due to the relative infrequency with which granulomata are found and the ambiguous nature of microgranulomata, variants or possibly precursors of true granulomata, pathologists have tried to use other features to make the distinction. These include crypt abscess formation, the distribution of the inflammation, gland atrophy and mucin depletion. Mucin depletion and gland atrophy have been the classic features used, ${ }^{5}$ and the aim of this study was to test the validity of these features.

Gastroenterology A S Mee

Royal Berkshire Hospital, Reading

Correspondence to: Dr Deirdre McCormick, Senior Registrar, Department of

Histopathology,

Queen Alexander Hospital Cosham, Portsmouth PO6 3LY, England.

Accepted for publication

11 September 1989 rectal biopsy specime with ulcerative colitis, and 11 from patients with Crohn's disease. The conclusive categorisation of patients was made by a gastroenterologist on the basis of all the information available from clinical assessment, endoscopy, radiology and pathology. The group with ulcerative colitis was selected from 68 rectal biopsy specimens, which showed active inflammation suggestive of ulcerative colitis. These cases were extracted from the pathology files for the years 1986 and 1987. Some of these were excluded because the patients had only a single episode of colitis, others because subsequent biopsy specimens were diagnostic of Crohn's disease, and in some cases the colitis was related to drugs. In a large number of cases there remained some doubt about the final diagnosis. The 10 cases finally selected were of adequate size and had a firm diagnosis of ulcerative colitis.

The group with Crohn's disease were selected from 40 established cases on file in the gastroenterology department. Eleven of these cases provided specimens of sufficient size to be included in the study. All of these were acutely inflamed and six also contained granulomata.

\section{IMAGE ANALYSIS}

A Quantimet 900 (Cambridge Instruments) image analysis system was used to make the measurements. An adequate distinction between mucin containing glands was obtained only by the use of special stains so all the biopsy specimens were stained with alcian blue and periodic acid Schiff (fig 1). These were then viewed with a Reichert Jung Polyvar microscope using bright field illumination and a neutral density filter. The microscope was set at a $\times 10$ objective giving a field size of $2.4 \mathrm{~mm}$. Using a grid in one eyepiece, two areas $\left(1 \mathrm{~mm}^{2}\right)$, each of which was completely covered by mucosa, were assessed on every biopsy specimen.

A video camera picked up the image and displayed it on a screen. The computer was set to register mucin stained with alcian blue and periodic acid Schiff so that mucin containing cells registered as light grey in contrast to non-mucin containing cells which were darker grey. The threshold was set for optimal sensing of mucin by comparing mucin in the microscopic image with that on the video display unit. Even then there were residual false positive areas which had to be edited out. The total epithelial area was registered by tracing around the luminal surface and the basement membrane separating the epithelium from the lamina propria, using a light pen to interact with the screen image. The following measurements 


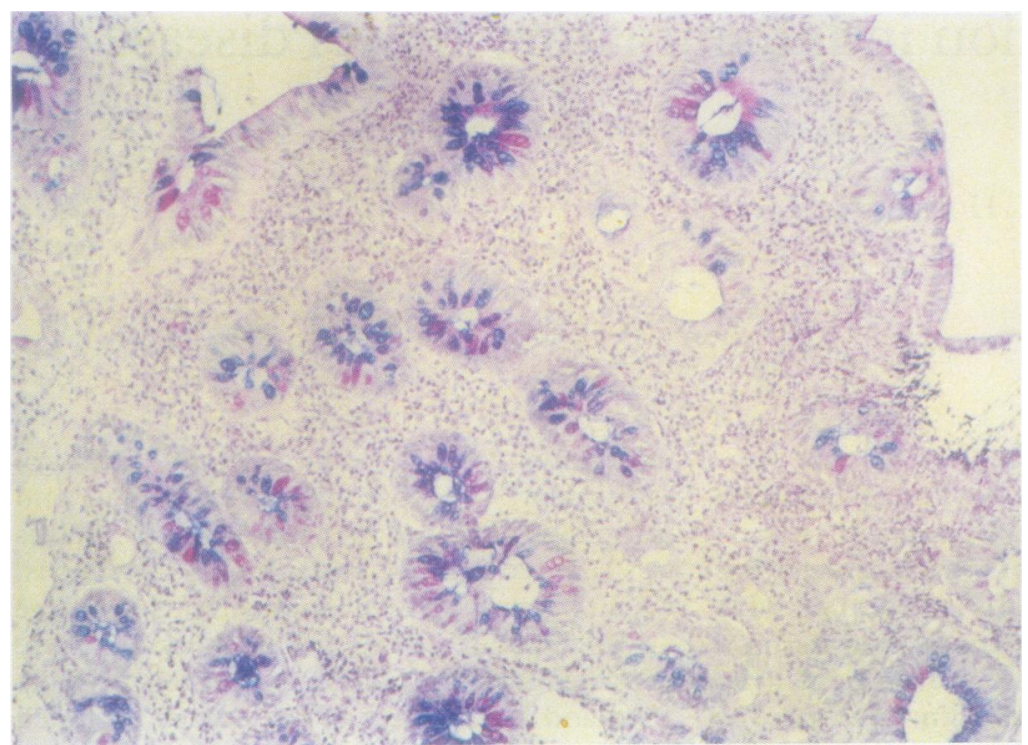

Figure 1 Rectal biopsy specimen from a patient with ulcerative colitis

showing mucin depletion (Alcian blue and periodic acid Schiff).

were made by the computer and expressed as percentages (fig 2).
Figure 2 Line drawing showing variables measured.

$$
\begin{aligned}
\text { Mucin content } & =\frac{\text { Total mucin area }}{\text { Total epithelial area }} \\
\text { Glandular content } & =\frac{\text { Glandular epithelial area }}{\text { Mucosal area }}
\end{aligned}
$$

The results were analysed using Wilcoxon's rank sum test for non-parametric data.

\section{Results}

MUCIN CONTENT

There was significantly less mucin in the group with ulcerative colitis (median 16.4\%) compared either with the normal group (median $42.8 . \%$ ) $\mathrm{p}<0.01$, or the group with Crohn's disease (median 38.7\%) $\mathrm{p}<0.01$.
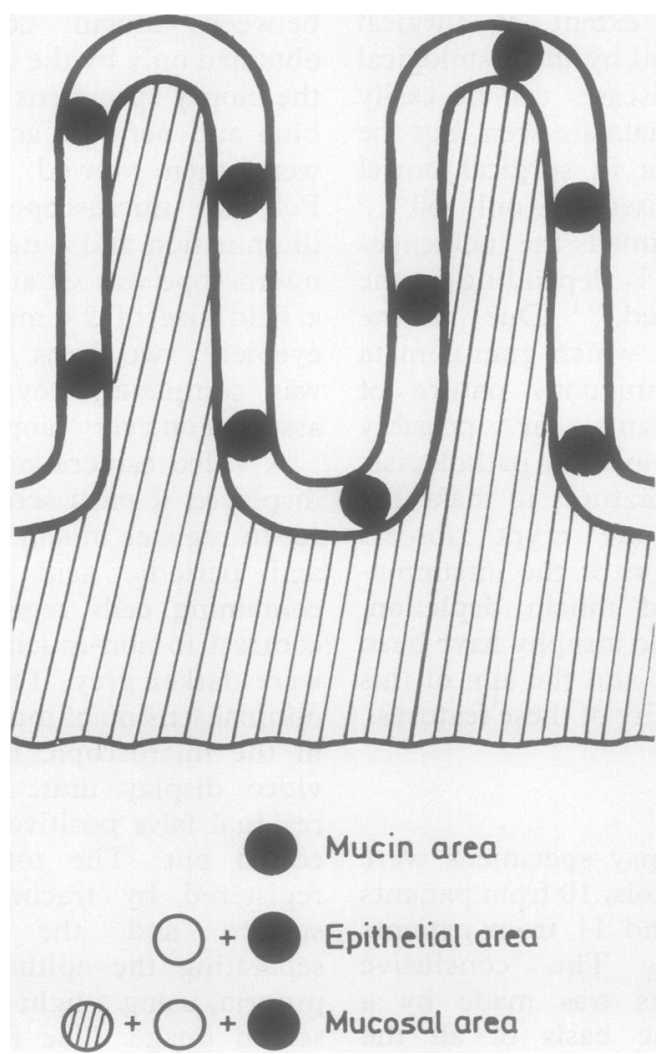

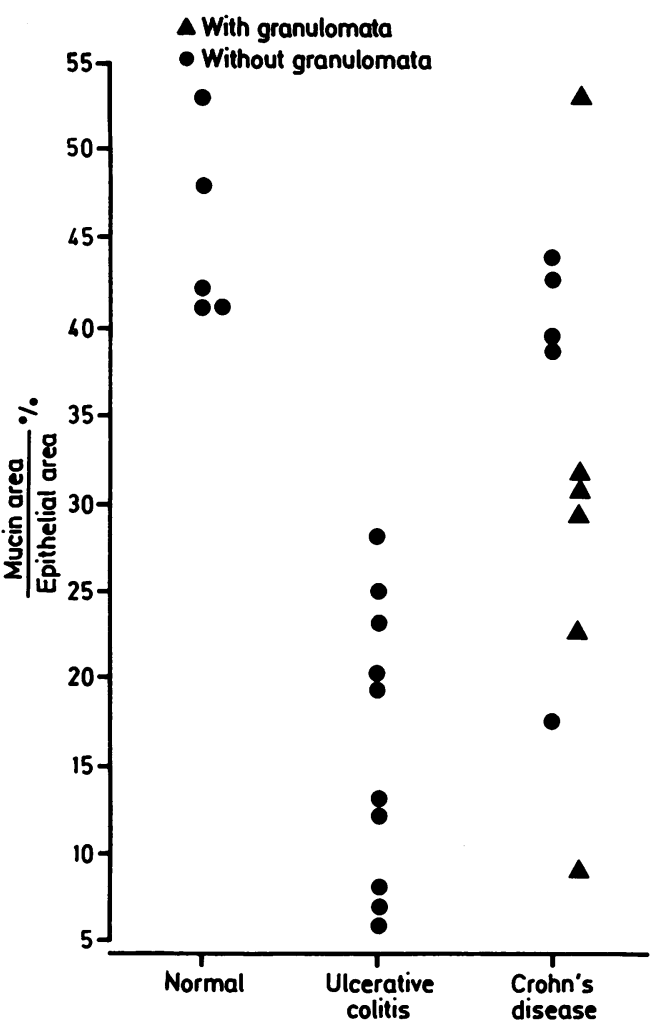

Figure 3 Mucin area expressed as percentage of epithelial area.

There was no significant difference in the mucin content between the group with Crohn's disease and the normal controls (fig 3).

\section{GLAND CONTENT}

There was a significant difference in the mucosal gland content between the group with ulcerative colitis (median 56.1\%) and the normal controls (median $76.9 \%$ ) $p<0.05$. The differences in the mucosal gland content

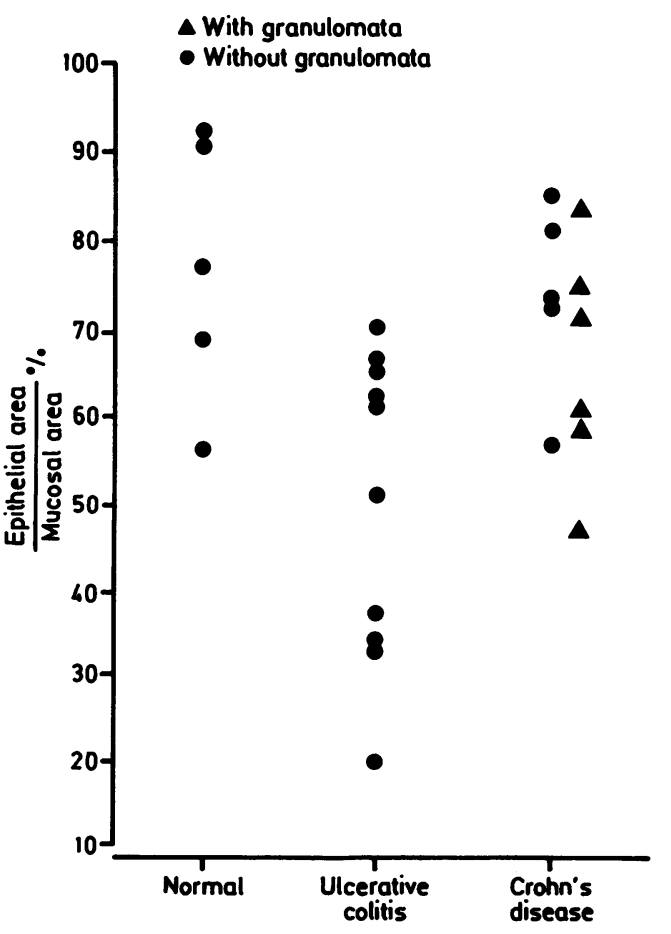

Figure 4 Epithelial area expressed as percentage of mucosal area. 
between the two disease groups and between the group with Crohn's disease (median $72 \cdot 7 \%$ ) and normal controls did not reach significance (fig 4).

\section{Discussion}

There has long been controversy about the relative importance of mucin depletion and gland atrophy as criteria for distinguishing between ulcerative colitis and Crohn's disease. Hellstrom and Fisher estimated the mucosal mucin in colectomy specimens from patients with Crohn's disease and ulcerative colitis. ${ }^{6}$ They found a striking difference in the mucosal content in these disorders. In chronic ulcerative colitis it was noticeably decreased or only partially preserved while the mucosal mucin content was well preserved in Crohn's disease. Their patients all had surgery and findings were therefore biased towards severity, as opposed to the cases in our study, which were rectal biopsy specimens taken from patients who had active disease. These authors concluded that estimation of the mucosal mucin content allowed the distinction between ulcerative colitis and Crohn's disease to be made confidently.

In a study which applied numerical taxonomy and discriminant analysis to nonspecific colitis the authors found that the disorder termed proctocolitis comprised a single relatively homogeneous group of patients whereas colonic Crohn's disease was a more heterogeneous group composed of distinguishable subgroups. ${ }^{7}$ Most of the distinctions between the main computer groups were based on five attributes, and the one which gave the greatest discrimination was the histological criterion of mucin depletion. The mucin content of goblet cells was reduced in proctocolitis but was often normal in Crohn's disease despite severe inflammatory cell infiltration in the lamina propria. Other studies however, have, challenged these views. ${ }^{189}$

One such opposing view was held by Sommers and Korelitz following a study of mucosal cell counts in ulcerative colitis and granulomatous colitis. ${ }^{9}$ These authors found significantly decreased mucous goblet cells both in sigmoidoscopically abnormal mucosa in ulcerative colitis and in granulomatous colitis and noted that they increased in the healing process. Mucin can be assessed either by counting goblet cells as done by Sommers and Korelitz or by making an overall evaluation of the mucin content of the glandular epithelium as in our study. We consider that the latter measurement is a more meaningful way of assessing mucin content because the mucin content of each individual goblet cell may vary, and therefore counting these cells may either overestimate or underestimate the amount of mucin.

Similarly, Surawicz et al found that half of their patients with Crohn's disease showed depletion of goblet cell mucus in a study of the value of rectal biopsy in the diagnosis of Crohn's disease. They concluded that mucus depletion was a non-specific finding and probably reflected the degree of surrounding inflammation. In their assessment, however, mucus depletion was defined as an obvious decrease in the amount of mucus within goblet cells and measured subjectively by the observer's eye, and not by an image analyser as in our study.

In a more recent study Thompson et al assessed mucin depletion along with other variables in rectal biopsy specimens from patients with inflammatory bowel disease. ${ }^{8}$ These authors used computed interactive image analysis to make their measurements and the results were analysed by forward stepwise discriminant analysis. Mucin depletion and crypt length were found to be poor discriminators of types of inflammatory bowel disease. The ulcerative colitis study group, however, included both patients with active disease and disease in remission, compared with patients in our study from whom only biopsy specimens showing active disease were included, and this may account for the differing results.

While the role of mucin depletion as a discriminating factor between ulcerative colitis and Crohn's disease has been examined, others have turned their attention to the actual mechanism of mucin depletion in inflammatory bowel disease. This has not been clearly defined but several interesting findings have been reported. In active ulcerative colitis the epithelial cell turnover has been shown to rapidly increase with upward expansion of the crypt proliferation zones $^{1011}$ resulting in exfoliation of immature cells ${ }^{12}$ and discharge of mucus prematurely before the completion of the mucus synthesis. ${ }^{13}$

Qualitative changes in colonic mucus have also been reported: these include a consistent deficiency in one particular mucus species in both active and inactive ulcerative colitis. This finding points to an underlying structural change in colonic mucin that may be of relevance to the pathogenesis of the disease. ${ }^{14}$

Assessment of the glandular content was not a useful feature in our study for distinguishing between ulcerative colitis and Crohn's disease. The gland content of the biopsy specimens was expressed per unit area as opposed to per unit length of muscularis mucosae. This method was chosen because this was a retrospective study, and therefore not all the biopsy specimens were properly orientated to allow the measurements to be made per unit length of muscularis mucosae. This method only detects disproportionate gland atrophy. Our study did not show any significant difference in the gland atrophy detected in the ulcerative colitis as opposed to Crohn's disease, and this agrees with the findings of Thompson et al. ${ }^{8}$

It is obviously not practical in routine reporting to quantitate accurately the amount of mucin in rectal biopsy specimens, but it is possible to assess the mucin content roughly by eye as normal, mild, moderately or severely 
depleted. This assessment can be facilitated by staining one section with alcian blue and periodic acid Schiff. Mucin content must of course be taken in conjunction with other features such as the pattern of inflammation and crypt abscess formation when attempting to distinguish between ulcerative colitis and Crohn's disease on rectal biopsy material in the absence of granulomata. This study supports the value of assessing the mucin content of these biopsy specimens when faced with this diagnostic problem.

We are very grateful to $\mathrm{Mr} \mathrm{J}$ Hicklin and Miss $\mathrm{J}$ Heathcock from The Lord Zuckerman Research Centre, Reading University, for their invaluable assistance with the image analysis. We also thank $\mathrm{Mr} \mathrm{J}$ Martyn for typing the manuscript and Mr R McLean for photography.

1 Surawicz CM, Meisel JL, Ylvisaker T, Saunders DR, Rubin CE. Rectal biopsy in the diagnosis of Crohn's disease: value of multiple biopsies and serial sectioning. Gastroent erol 1981;81:66-71.

2 Morson BC. Systemic pathology. In: Alimentary tract
Edinburgh: Churchill Livingstone, 1987:258.

3 Petri M, Poulsen SS, Christensen K, Jarnum S. The incidence of granulomas in serial sections of rectal biopsies from patients with Crohn's disease. Acta Pathol Microbio Immunol Scand (Sect A) 1982;90:145-7.

4 Rotterdam H, Korelitz BI, Sommers SC. Microgranulomas in grossly normal rectal mucosa in Crohn's disease. $\mathrm{Am}$ Clin Path 1977;67:550-4.

5 Morson BC. Rectal biopsy in inflammatory bowel disease. $N$ Engl J Med 1972;287:1337-9.

6 Hellstrom HR, Fisher ER. Estimation of mucosal mucin as an aid in the differentiation of Crohn's disease of colon and an aid in the differentiation of Crohn's disease of colon and 259-68.

7 Hywel Jones J, Lennard-Jones JE, Morson BC, et al. Numerical taxonomy and discriminant analysis applied to non specific colitis. $Q J$ Med 1973;42:715-32.

8 Thompson EM, Price AB, Altman DG, Sowter C, Slavin G. Quantitation in inflammatory bowel disease using computerised interactive image analysis. $J$ Clin Pathol 1985 38:631-8.

9 Sommers SC, Korelitz MD. Mucosal cell counts in ulcerative and granulomatous colitis. Am J Clin Pathol 1975;63:359-65.

10 Eastwood GL, Trier JS. Epithelial cell renewal in cultured rectal biopsies in ulcerative colitis. Gastroenterol 1973; 64:383-90.

11 Kanemitsu T, Koike A, Yamamoto S. Study of the cell proliferation kinetics in ulcerative colitis, adenomatous polyps, and cancer. Cancer 1985;56:1094-8.

12 Allan A, Bristol JB, Williamson RCN. Crypt cell production rate in ulcerative proctocolitis: differential increments in remission and relapse. Gut 1985;26:999-1003.

13 Cope GF, Heatley RV, Kelleher J, Axon ATR. In vitro mucus glycoprotein production by colonic tissue from patients with ulcerative colitis. Gut 1988;29:229-34.

14 Podolosky DK, Isselbacher KJ. Glycoprotein composition of colonic mucosa. Gastroenterol 1984;87:991-8. 